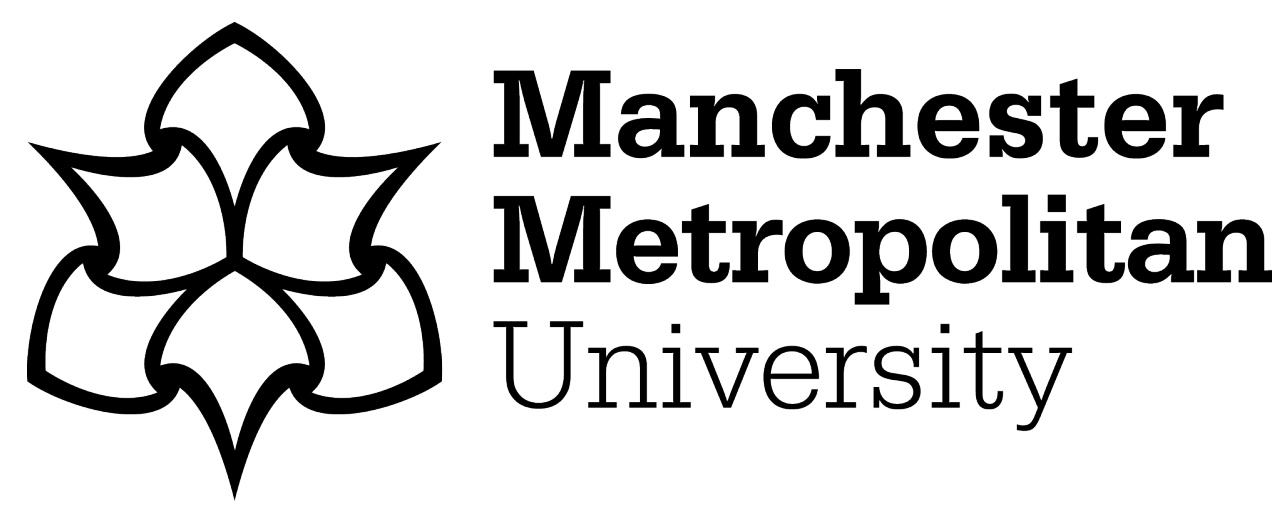

Bruce, Julie, Mazuquin, Bruno ORCID logoORCID: https://orcid.org/00000003-1566-9551, Mistry, Pankaj, Williamson, Esther, Lall, Ranjit, Vidya, Raghavan, Withers, Emma, Rees, Sophie, Canaway, Alastair, Petrou, Stavros, Lamb, Sarah E and Thompson, Alastair M (2020) Prevention of shoulder problems trial (UK-PROSPER): Exercise to prevent shoulder problems in patients undergoing breast cancer treatment. In: 42nd Annual San Antonio Breast Cancer Symposium, 09 December 2019 - 14 December 2019, San Antonio, TX.

Downloaded from: https://e-space.mmu.ac.uk/628553/

Version: Accepted Version

Publisher: American Association for Cancer Research

DOI: https://doi.org/10.1158/1538-7445.SABCS19-P1-13-04

Please cite the published version 


\title{
Abstract P1-13-04: Prevention of shoulder problems trial (UK-PROSPER): Exercise to prevent shoulder problems in patients undergoing breast cancer treatment
}

Julie Bruce, Bruno Mazuquin, Pankaj Mistry, Esther Williamson, Ranjit Lall, Raghavan Vidya, Emma Withers, Sophie Rees, Alastair Canaway, Stavros Petrou, Sarah E Lamb and Alastair M Thompson

\begin{abstract}
Aim: To investigate the clinical and cost-effectiveness of early structured exercise on functional and health-related outcomes in women at high risk of developing shoulder problems after breast cancer treatment. Methods: The UK-PROSPER was a pragmatic, multicentre, randomised controlled trial comparing a structured exercise programme with best practice usual care (information leaflet). Women at high-risk (planned axillary node clearance, planned radiotherapy to the axilla and/or supraclavicular area, existing shoulder problems and BMI $>30$ ) of postoperative shoulder problems with a confirmed invasive or non-invasive primary breast cancer scheduled for surgery were included. We aimed to randomise 350 women from 17 centres across England, with postoperative follow-up at 6 weeks, 6 and 12 months postrandomisation. The PROSPER exercise intervention was developed from evidence, clinical experts and patients; the final intervention consisted of exercise, behavioural strategies and physical activity. Women were offered a minimum of three face-to-face appointments with a physiotherapist, starting 7 to 10 days postoperatively with three optional appointments. The control group received written leaflets containing information on exercises after surgery. The primary outcome was the Disabilities of the Arm Shoulder and Hand (DASH) score at 12 months. Secondary outcomes included DASH subscales, postoperative pain, complications and health-related quality of life. Results: 392 women were recruited, 196 randomised to each treatment arm. Mean age was 58.1 years, mean BMI was 30.2. Groups were similar for socioeconomic characteristics at baseline. Of 191 women allocated to exercise intervention, $142 / 191(74 \%)$ received three or more PT sessions, 38/191 (20\%) received up to three sessions and 11/191 (6\%) no treatment. Those randomised to exercise had better arm, shoulder and hand function compared to usual care at 12 months (mean DASH score 16.4 versus 23.9 respectively; adjusted mean

difference (MD) -7.42 ; 95\% CI -12.34 to $-2.50 ; \mathrm{p}=0.003$ ). DASH subscales for activity limitations, participation restriction and impairment improved over time in women receiving exercise compared to usual care. A lower rate of neuropathic pain at 12 months was reported by treatment arm (23\% control vs 16\% exercise). Conclusions: Early structured postoperative exercise was beneficial on functional and other health-related outcomes at 12 months in women undergoing surgery for breast cancer treatment. Early postoperative exercise was safe and did not increase risk of postoperative adverse events
\end{abstract}

